\title{
Planar Rectangular Sets and Steiner Symmetrization
}

\author{
Paul R. Scott
}

Paul Scott has worked in the Department of Pure Mathematics at the University Adelaide for the past 30 years. His research interests are in convex sets and the geometry of numbers. On the teaching side, he is experimenting with computer presentation of lectures and computer aided learning. He is also interested in mathematics education, and has edited and typeset 'The Australian Mathematics Teacher' for the past seven years. His outside interests include travel, photography, classical music and six grandchildren.

\section{Introduction}

Let $K$ be a closed convex set in the plane. In [1], Danzer establishes the following pretty result.

Theorem 1. If no rectangle inscribed in $K$ has exactly three of its vertices on the boundary of $K$, then $K$ is a circular disk.

We generalize Danzer's characterization in the following way. Let $O X$, OY be given, fixed orthogonal axes in the plane. We say that $K$ is a rectangular set if no inscribed rectangle with edges parallel to the given axes has exactly three of its vertices on the boundary of $K$. Some anomalies can occur in this new setting. For example, if $K$ has two adjacent perpendicular edges which are parallel to the axes, there is an infinite number of 'inscribed' rectangles having just three vertices on the boundary of $K$. We therefore interpret inscribed here to imply that the given rectangle is the largest in the family of homothetic rectangles having vertices on the boundary of $K$. This is the assumption we would make if talking about an incircle of $K$.

We now ask if it is possible to characterize in some way the family $\mathscr{R}$ of rectangular sets. We note that $\mathscr{R}$ contains sets which are symmetric about either or both of the axes.

Let $K$ be a closed convex set in the plane, and $O X$, $O Y$ given, fixed orthogonal axes. We say that $K$ is a rectangular set if no inscribed rectangle with edges parallel to the given axes has exactly three of its vertices on the boundary of $K$. We show that if $S_{X}$, $S_{Y}$ denote Steiner symmetrizations about the axes $O X$, $O Y$ respectively, then $K$ is a rectangular set (relative to these axes) if and only if $S_{X} S_{Y}(K)=S_{Y} S_{X}(K)$. psc 
It turns out that the family $\mathscr{R}$ has a nice characterization in terms of Steiner symmetrization, which we now define. Let $O A$ be a given line - the axis $l$ of symmetrization. For each point $p$ on $O A$ let $u(p)$ be the line through $p$ which is perpendicular to $l$. The set $u(p) \cap K$ is either the empty set, a point, or a line segment. If it is the empty set, we define $B(p)$ to be the empty set. If it is a point, we define $B(p)$ to be the point $p$. If it is a line segment, we define $B(p)$ to be the segment of equal length whose midpoint is $p$ and which lies on $u(p)$. We now define $K_{A}$ by

$$
K_{A}=\cup_{p \in l} B(p) .
$$

The process of obtaining $K_{A}$ from $K$ in this way is called Steiner symmetrization about the line $O A$. Properties of this well-known and useful form of symmetrization can be found, for example, in Eggleston [2].

We shall establish the following connection between Steiner symmetrization and the family $\mathscr{R}$ of rectangular sets.

Theorem 2. Let $S_{X}, S_{Y}$ denote symmetrizations about the axes OX, OY respectively. Then $K$ is a rectangular set (relative to these axes) if and only if

$$
S_{X} S_{Y}(K)=S_{Y} S_{X}(K)
$$

\section{Proof of Theorem 2}

For consistency in naming in the proof, we drop the function notation used in the statement of the theorem, and use $S_{X} S_{Y}$, for example, to mean first apply $S_{X}$ and then apply $S_{Y}$. We shall also use the words horizontal and vertical to describe lines which are parallel to $O X, O Y$ respectively.

First we suppose that $K$ is a rectangular set. Let $A$ be a point on the boundary of $K$. By assumption, $A$ will be a vertex of a (perhaps degenerate) rectangle $A B C D$ whose four vertices lie on the boundary of $K$ (see Figure 1$)$.
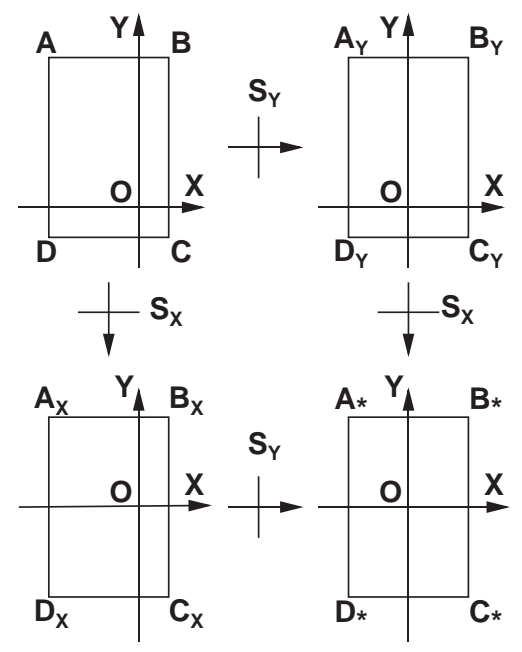

Fig. 1 
Let $A B=2 x$ and $B C=2 y$. If we symmetrize $K$ using $S_{Y}$ to obtain a symmetrized set $K_{Y}$, then $A$ will map to a point $A_{Y}$, a vertex of a rectangle $A_{Y} B_{Y} C_{Y} D_{Y}$, inscribed in $K_{Y}$, and congruent to $A B C D$. For, under the symmetrization, lengths $A B, D C$ are preserved, and the image segments $A_{Y} B_{Y}, D_{Y} C_{Y}$ are centred on the axis $O Y$. In particular, $A_{Y}$ has $x$-coordinate $x$, and $A_{Y} D_{Y}=2 y$. If we now symmetrize $K_{Y}$ using $S_{X}$ to obtain set $K_{Y X}$, then $A_{Y}$ maps to a point $A_{Y X}$, a vertex of a rectangle inscribed in $K_{Y X}$ and congruent to $A B C D$. For, under the symmetrization, lengths $A_{Y} D_{Y}, B_{Y} C_{Y}$ are preserved, and the image segments $A_{Y} D_{Y}, B_{Y} C_{Y}$ are centred on the axis $O X$. In particular, $A_{Y X}$ has $x$-coordinate $x$, and $y$-coordinate $y$.

It is clear from the symmetry of $X$ and $Y$ in this argument that the image of $A$ under the product $S_{X} S_{Y}$ will be $A_{X Y}=A_{Y X}\left(=A_{*}\right.$ in Figure 1). We deduce that $K_{X Y}=K_{Y X}$.

Now let us suppose that $K$ is a set which has the same image under $S_{Y} S_{X}$ as it does under $S_{X} S_{Y}$. Thus $K_{Y X}=K_{X Y}$. We wish to show that $K$ is a rectangular set. We observe that it will be sufficient to establish this result for the case when $K$ is a polygon. The general case will then follow using a standard approximation argument. We may thus assume that the final symmetrized set $K_{X Y}=K_{Y X}$ is the convex hull of a finite family of rectangles having horizontal and vertical edges. If each of these rectangles occurs as the image of an inscribed rectangle in $K$, then $K$ is a rectangular set, and there is nothing to prove. Suppose then that one of these rectangles, $R_{X Y}=R_{Y X}$ does not occur in this way. Let this rectangle have horizontal and vertical dimensions $2 x, 2 y$ respectively. Suppose too that $y$ is the largest number for which this happens.

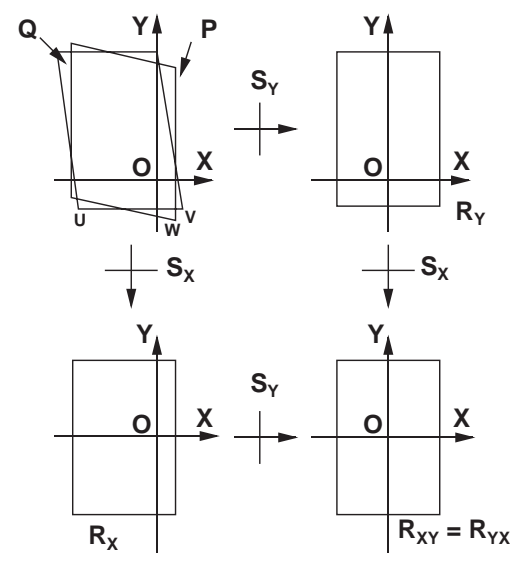

Fig. 2

Now $R_{X Y}$ is the image under $S_{Y}$ of a set $R_{X}$ (see Figure 2). In fact $R_{X}$ is itself a rectangle, since it is inscribed in a set $K_{X}$ which is symmetric about the $X$-axis. Further, $R_{X}$ has horizontal and vertical dimensions $2 x, 2 y$ respectively. Now rectangle $R_{X}$ occurs as the image under symmetrization $S_{X}$ of a set $P$ inscribed in the original set $K$. By the properties of symmetrization, this set $P$ must be a parallelogram having one pair of vertical parallel edges. The length of each of these parallel edges is $2 y$, and the 
distance between them is $2 x$. In the same way, $R_{X Y}$ occurs as the image under $S_{Y} S_{X}$ of a parallelogram $Q$ inscribed in $K$ having two horizontal parallel edges; the length of each of these parallel edges is $2 x$, and the distance between them is $2 y$.

If either of $P, Q$ is a rectangle, then $P, Q$ will coincide, as we have already seen that the image of a rectangle inscribed in $K$ having horizontal and vertical edges is the same under the two successive symmetrizations, no matter which order of symmetrization is used. Hence parallelogram $P$ extends strictly above or below the parallel horizontal edges of parallelogram $Q$. Inverting the figure if necessary, we may assume that $P$ extends strictly below $Q$. Let $U V$ denote the bottom horizontal edge of $Q$, labelled as in Figure 2, and $W$ the vertex of $P$ which lies below it. Then points $U, W, V$ lie in an anti-clockwise order on the boundary of $K$. Since symmetrization is a continuous transformation, $U, W, V$ will map under the successive symmetrizations $S_{Y}, S_{X}$ to image points $U^{*}, W^{*}, V^{*}$ lying in anti-clockwise order on the boundary of $K_{X Y}$. But $U^{*} V^{*}$ is the bottom edge of $R_{X Y}$. It follows that $W^{*}$ is the vertex of a rectangle inscribed in $K_{X Y}$ which does not arise as the image of a rectangle inscribed in $K$. Further, the vertical dimension of this rectangle exceeds the vertical dimension $2 y$ of $R_{X Y}$ which was chosen to be maximal. This contradiction establishes the theorem.

\section{Final Comment}

The class of rectangular sets appears naturally here in terms of successive orthogonal symmetrizations; to my knowledge, this class does not occur elsewhere in the literature. It would be interesting to investigate whether this class of sets has other special properties.

\section{References}

[1] Danzer, L. W., "A characterization of the circle", Proc. Symp. Pure. Maths VII (1963), 99-100.

[2] Eggleston, H. G., Convexity, Cambridge Tract No. 47 (1963), Cambridge University Press.

Paul R. Scott

University of Adelaide

Adelaide

South Australia 